\section{An Audit on Operation Note Documentation in the Bristol Royal Infirmary ENT department}

Dr Charlotte Thomas, Dr Lucy Lee-Warder, Mr James Morris. Bristol Royal Infirmary ENT department
University Hospitals

Bristol

NHS Foundation Trust

"Surgeons must ensure that accurate, comprehensive, legible and contemporaneous records are maintained of all their interactions with patients." Good Surgical Practice 20141. Careful operation note documentation is essential to ensure continuity of care between the operating team and other colleagues, as well as providing a medicolegal record of patient care.

\section{AlMS}

1. To review the documentation of operation notes within the adult and paediatric ENT departments in the Bristol Royal Infirmary (BRI) and compare this to the standard recommended by the Royal College of Surgeons.

2. To ensure awareness of these good practice guidelines in the ENT department and to implement a new operation note proforma for use.

\section{METHOD}

To audit 20 recent operation notes and compare these to the guideline set by the royal college of surgeons. From this sample to identify problems areas and address these by making a change in the operation note format. Once the new operation notes were in circulation to re-audit 20 more operation notes and see if our intervention was successful or if further changes could be made.

\title{
RESULTS
}

20 operation notes written between 25/10/17 - 08/11/17 were reviewed. None of the operation notes reviewed documented whether the operation had been done in an emergency or elective setting or the time it occurred, just $25 \%$ documented whether prophylactic antibiotics were required and $60 \%$ documented whether VTE prophylaxis was required post operatively. The changes we made to the operation note are shown in red in Figure 1. Following these few simple changes 20 operation notes were again reviewed between 11/1/18 and 18/1/18. There were significant improvements in operation note documentation with time and elective/emergency now being recorded in $90 \%$, antibiotic prophylaxis in $80 \%$ and VTE prophylaxis in $80 \%$. Documentation before and after implementation on the new operation note shown in Figure 2.

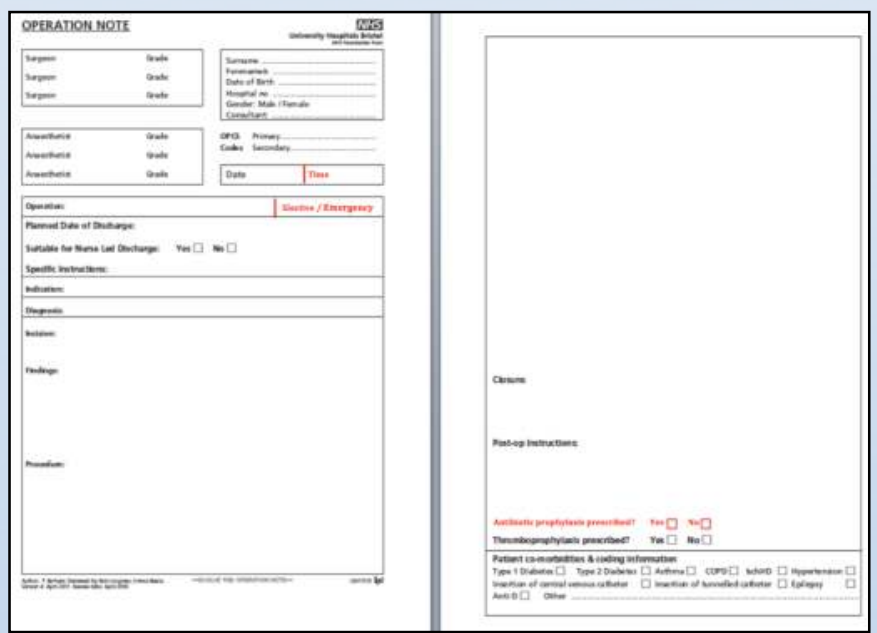

Figure 1. Original operation note with changes highlighted in red.

\section{CONCLUSIONS}

Our intervention to alter the operation note has successfully improved documentation of time, elective or emergency, VTE and antibiotic prophylaxis and this will have a positive impact on patient safety. The increase in VTE prophylaxis and complication documentation even though no intervention was made, was perhaps due to surgeon awareness of operation note changes.

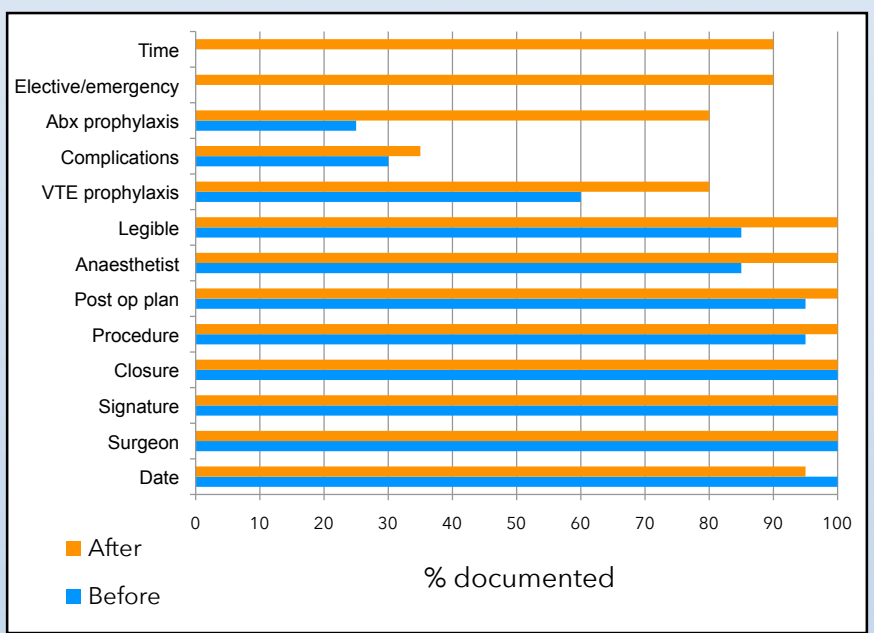

Figure 2. Bar chart showing documentation before and after the new operation note.

\section{FURTHER WORK}

We plan to re-audit in the next few months to see if documentation rates remain improved.

We are also working with one of our ENT consultants who is introducing electronic operation notes to the trust - as despite our $100 \%$ record of legibility - this is surgeon dependent and is a common issue highlighted by the theatre team in recovery. 